\title{
A hybrid technique of combined conventional and robotic-assisted laparoscopy for staging and debulking of early, advanced, and recurrent ovarian, fallopian tube, and primary peritoneal cancer
}

\author{
Farr R. Nezhat • Susan S. Khalil • Tamara N. Finger • \\ Patrick F. Vetere
}

Received: 24 June 2013 / Accepted: 2 September 2013 / Published online: 5 November 2013

(C) Springer-Verlag Berlin Heidelberg 2013

\begin{abstract}
This study aims to describe a hybrid laparoscopic and robotic-assisted laparoscopic technique to access all four abdominal quadrants during pelvic procedures. This technique was utilized in the surgical management of select cases that included early, advanced, and recurrent ovarian, fallopian tube, and peritoneal cancer. A retrospective analysis of a prospectively maintained database was used to extract cases that this surgical method was utilized in. This included 20 patients that underwent 21 surgical procedures using this hybrid technique of conventional laparoscopy and robotic-assisted laparoscopy. Ten were early stage, and 11 were advanced and/or recurrent (six advanced, five recurrent). In the early-stage group, mean age was 42.3 years (range, 29-55), average BMI was $32.1 \mathrm{~kg}$ / $\mathrm{m}^{2}$ (range, $17-65 \mathrm{~kg} / \mathrm{m}^{2}$ ), mean blood loss was $212.5 \mathrm{ml}$ (range,
\end{abstract}

\section{F. R. Nezhat}

Division of Gynecologic, Oncology and Minimally Invasive Surgery, Department of Obstetrics and Gynecology, Columbia University, New York, NY 10019, USA

\section{F. R. Nezhat $(\triangle)$}

Department of Obstetrics and Gynecology, St. Luke's-Roosevelt Medical Center, 10th Floor, 1000 10th Avenue, New York, NY 10019, USA

e-mail: fnezhat@chpnet.org

F. R. Nezhat

e-mail: frn2103@columbia.edu

F. R. Nezhat $\cdot$ S. S. Khalil $\cdot$ T. N. Finger

Division of Minimally Invasive Surgery, Department of Obstetrics and Gynecology, St. Luke's-Roosevelt Medical Center, New York, NY, USA

F. R. Nezhat • P. F. Vetere

Division of Minimally Invasive Gynecologic Surgery, Department of Obstetrics and Gynecology, Winthrop University Hospital, Mineola, NY, USA
50-1,000 mL), operating room time (ORT) was $306.1 \mathrm{~min}$ (range, 87-639), and average length of stay (LOS) was 1.6 days (range, 1-2). There were no intraoperative complications and two grade 1 postoperative complications. Of the 11 for advanced and/or recurrent disease, mean age was 63.9 years (range, 39-92), average BMI was $29.7 \mathrm{~kg} / \mathrm{m}^{2}$ (range, 22.137.2), mean blood loss was $129.1 \mathrm{ml}$ (range, 20-400), ORT was 238 min (range, 103-477), and LOS was 3.8 days (range, 117). There were no intraoperative complications. Three cases had postoperative grade 1-3 complications. There was one second look, nine cytoreductions to no visible disease, and 1 to $<0.5 \mathrm{~cm}$. Use of this hybrid technique, combining conventional laparoscopy and the present robotic platform, is effective in the surgical management of early, advanced and recurrent ovarian, fallopian tube, and peritoneal cancer in accessing all four abdominal quadrants with pelvic surgery.

Keywords Hybrid technique - Conventional laparoscopy · Robotic-assisted laparoscopy · Ovarian cancer Staging and Cytoreduction

\section{Introduction}

Advances in minimally invasive surgical techniques now make it feasible to accomplish comprehensive surgical staging, using conventional or robotic-assisted laparoscopy in select patients [1]. The present computer enhanced telesurgery (Intuitive Surgical, Sunnyvale, CA, USA), called robotassisted surgery, was approved by the Food and Drug Administration for gynecological surgery in 2005 and has been increasingly applied to complex gynecologic procedures, such 
as surgical staging for gynecologic malignancies including endometrial, cervical, and ovarian cancers [2-5].

Limitations exist with the present robotic platform for staging and cytoreduction in ovarian cancer. Once the robot is docked for pelvic surgery, it is more difficult to access the upper abdomen, without having to undock and reposition the robot, or add additional ports to be able to perform the procedure. The Society of Gynecologic Oncology's consensus statement on robotic-assisted surgery commented on its utility in ovarian cancer as poorly suited for advanced ovarian cancer due to its limitation, with conventional port placement for pelvic surgery, in gaining upper abdominal access [6].

In the USA, ovarian cancer will affect approximately 22,280 women in 2012 with 15,500 estimated deaths. Currently, the lifetime risk of developing ovarian cancer in the USA is approximately 1 in 70 with more than $65 \%$ given the diagnosis of advanced stage disease [7]. The standard treatment of ovarian cancer includes upfront surgery with intent to properly diagnose, stage, and to achieve maximal cytoreduction preferably to no visible disease followed by taxanes and platinum-based combination chemotherapy in majority of cases [8]. Traditionally, a comprehensive surgical staging procedure for ovarian, fallopian tube, and primary peritoneal cancers include total abdominal hysterectomy, bilateral salpingo-oophorectomy, peritoneal washings, biopsies of adhesions and peritoneal surfaces, omentectomy, and retroperitoneal lymph node sampling from the pelvic and para-aortic regions through a generous vertical midline laparotomy incision.

There have been attempts to strategize the utility of the robot for such cases in order to gain upper abdominal access without difficulty. Magrina et al. described their technique for approaching debulking procedures in patients with epithelial ovarian cancer that require upper abdominal access and for infrarenal aortic lymphadenectomy. This method involves undocking the robotic arms, then rotating the operating table $180^{\circ}$, insertion of additional ports, and then redocking [5, 9].

We describe our surgical method, a hybrid technique, in which both conventional laparoscopy and the robot are utilized in gynecologic malignancies in all four abdominal quadrants and the pelvic cavity. This surgical technique and its use in select patients with early and advanced ovarian cancer will be described for laparoscopic management of both pelvic and upper abdominal disease.

\section{Materials and methods}

A retrospective analysis of a prospectively maintained database was performed to extract select cases where this hybrid technique combining conventional laparoscopy (CL) and roboticassisted laparoscopic surgery (RALS) was utilized. These cases included early, advanced, and recurrent ovarian, fallopian tube, and peritoneal cancer. Institutional review board approval was obtained and data was collected from two urban university affiliated community hospitals. All patients underwent preoperative evaluation including history, physical examination, medical assessment, computed tomography imaging of the chest or abdomen and pelvis, or positron emission tomography scan, and tumor marker assays, and were counseled extensively preoperatively and appropriate informed consent was obtained. Patients with significant perioperative morbidity who were not candidates for any surgical procedures, either laparoscopy or laparotomy, or who had significant metastatic disease involving chest or solid organs, such as liver, were excluded. The same boardcertified gynecologist oncologist, assisted by a minimally invasive gynecological surgical fellow and resident performed the surgeries. Early stage disease included patients that were in stages I to II. This group also included patients referred for restaging after prior ovarian cystectomy or oophorectomy. Advanced disease was classified as International Federation of Gynecology and Obstetrics stages III to IV. Postoperative complications were graded using the Memorial Sloan-Kettering Cancer Center severity grading system [10].

\section{Technique}

Under general endotracheal anesthesia, the patients were positioned in dorsal lithotomy position, bilateral sequential compression devices were placed on both lower extremities and arms were padded and tucked. Egg crate foam was also placed across the chest to protect patients and they were secured to the operating table with tape or a gel pad underneath them. They were draped in a sterile manner, and given preoperative antibiotic prophylaxis with $1-2 \mathrm{~g}$ of cefazolin or $80 \mathrm{mg}$ or gentamycin and $900 \mathrm{mg}$ of clindamycin if they were allergic to penicillin. A Foley catheter was inserted into the bladder, and a uterine manipulator was placed if the uterus was in situ. An incision is made in either the left upper quadrant or $4-5 \mathrm{~cm}$ above the umbilicus, using a Veress needle to introduce carbon dioxide gas and establish pneumoperitoneum. After adequate pneumoperitoneum is obtained, a 5 or $8 \mathrm{~mm}$ primary port is inserted into the left upper quadrant. If pneumoperitoneum is established supraumbilically, then a 12-mm trocar and sleeve are introduced into the supraumbilical port. After assessing the abdominopelvic cavity, either a 10- or 12-mm port is introduced into the right upper quadrant or two $8-\mathrm{mm}$ robotic ports are introduced 8-10 cm lateral to the umbilicus bilaterally (Fig. 1). Further peritoneal inspection is performed by conventional laparoscopy and peritoneal washings or aspiration of existing ascites are obtained and sent for cytology. Decision to proceed with laparoscopic/robotic-assisted surgical or laparotomy staging or debulking was made based on the extent of the disease and patients comorbidity for lengthy operation. In advanced stages, the goal was to achieve cytoreduction to preferably no visible or at least $<1 \mathrm{~cm}$ disease either via robotic-assisted laparoscopy, conventional laparoscopy, or laparotomy. 


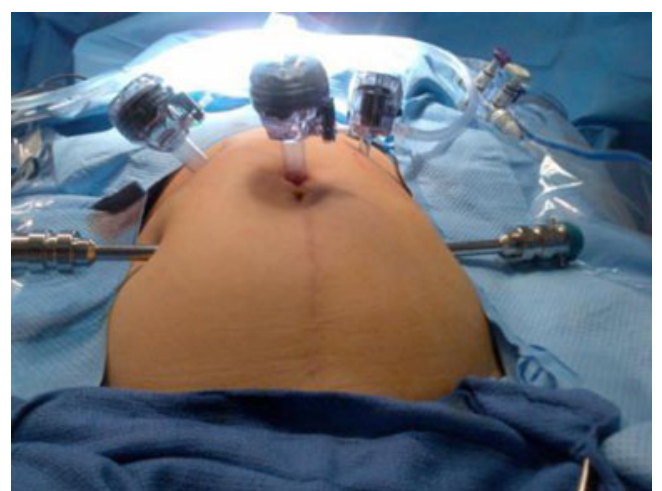

Fig. 1 Port placement

Thorough four-quadrant abdominopelvic cavity evaluation was performed by inserting additional ports, when necessary, to implement the choice of treatment. If surgical debulking to no visible disease is not possible, then biopsies are taken and salpingo-oophorectomy is performed if feasible $[11,12]$. The procedure is terminated and the patient is given neoadjuvant chemotherapy to achieve higher rate of optimal cytoreductive surgery and decrease morbidity [13]. The patient is then reoperated on after reducing the load of the disease, which has been shown in randomized trials not to compromise oncological outcomes. Only patients in whom this hybrid technique was utilized were included in this study.

If disease is present in the upper abdomen and pelvis, surgery begins with conventional laparoscopy to perform an omentectomy and upper abdominal debulking (Fig. 2). This is performed via use of the supraumbilical port for the camera and ports for introduction of instruments. The surgeon stands between the patient's legs with two assistants on either side of the
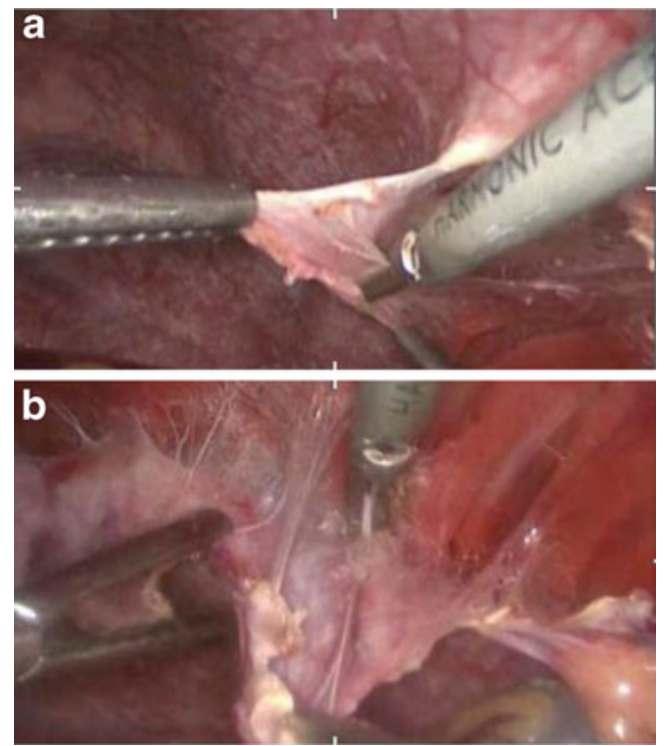

Fig. 2 a Diaphragmatic stripping of peritoneum with grasper and Harmonic scalpel (Ethicon Endosurgery, Cincinnati, OH, USA). b Diaphragmatic stripping showing stripped-away peritoneum and the underlying muscle fibers patient and an additional monitor placed towards the patient's head. A combination of various conventional laparoscopic instruments such as the Harmonic shears, the 5- or 10-mm LigaSure $^{\mathrm{TM}}$ (Covidien, Boulder, CO) or other blood vessel sealant devices including surgical clips and staples can be used. The infracolic omentum is transected from the transverse colon and the gastrocolic omentum is transected all the way towards the spleen and stomach by coagulating and transecting the short gastric vessels. The specimen(s) is removed confined to a laparoscopic bag. Metastatic lesions noted on the hepatic flexure or transverse colon are mobilized. Using a combination of Harmonic shears (Ethicon Endo-Surgery, Cincinnati, OH, USA), PlasmaJet (Plasma Surgical Limited, Oxfordshire, UK), and bipolar electrocoagulation, all diaphragmatic lesions are removed in the form of stripping, ablation, and coagulation (Fig. 2a,b). This same approach can be applied if there is no upper abdominal disease and only infracolic omentectomy is performed as part of surgical staging for presumed early ovarian cancer.

Any abdominal and pelvic adhesions which interfere with proper application of the robotic platform are lysed using conventional laparoscopy. After the upper abdominal portion is performed, the robotic apparatus is side docked on the patient's left side, using the supraumbilical port for the camera and the bilateral robotic $8-\mathrm{mm}$ ports. The posterior parietal peritoneum over the right common iliac is incised and retroperitoneal dissection is completed cephalad to above inferior mesenteric artery. We use the electrosurgical spatula or scissors as a cutting modality and bipolar forceps for achieving hemostasis. The left and right upper assist ports are utilized for introduction of ancillary instruments for traction, tissue removal, as well as suction and irrigation. After para-aortic lymphadenectomy is completed, pelvic lymphadenectomy, hysterectomy, bilateral salpingo-oophorectomy, and any pelvic tumor debulking are performed (Fig. 3). The same

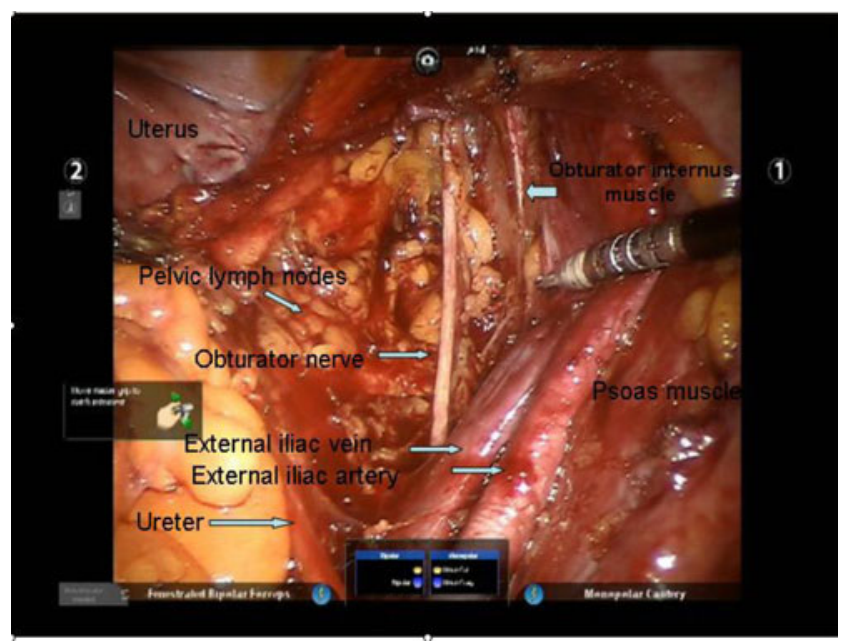

Fig. 3 Right pelvic sidewall exposing the iliac vessels and obturator fossa for robotic-assisted laparoscopic pelvic lymphadenectomy 
instrumentation utilized is as before, or at times, standard blood vessel sealing devices such as the LigaSure(Covidien, Boulder, CO, USA), etc. can be employed for ligation of the infundibular ligaments and performing hysterectomy. If access to the para-aortic lymph nodes above the inferior-mesenteric artery is not possible using the robotic platform setup, this portion of the operation is performed using a conventional laparoscopic approach after undocking the robot or the camera is moved from supraumbilical port to the right upper quadrant one. After the uterus is transected, it is removed from the vagina along with the omentum and any other specimens, which are confined to a laparoscopic specimen bag. The vaginal cuff is closed in two layers. After complete hemostasis is achieved, the robotic apparatus is undocked.

Bowel resections can be performed with conventional laparoscopy and robotically using the appropriate port and robot placement. In case of the need for mid-abdominal debulking, such as appendectomy or ileocecal resection, mobilization of the bowel is performed using the robotic platform, and appendectomy or bowel resection is performed using a stapling device introduced in the right upper abdominal port. Anastomosis can be performed either in situ or extracorporeally by extending the supraumbilical incision after pelvic tumor debulking and undocking the robot. This approach can be utilized for segmental transverse colon resection and reanastomosis to achieve optimal cytoreduction to no visible disease. For rectosigmoid colon resection and anastomosis, we use a $12-\mathrm{mm}$ port in the right lower abdomen for introduction of the stapling device. This is especially true for a bulky lesion involving the rectosigmoid colon. Using a laparoscopic $60 \mathrm{~mm}$ GIA stapler, a rectosigmoid resection can be performed proximally and distally. Once the proximal sigmoid colon is appropriately mobilized, this end can be brought out through a widened incision in the right lower quadrant, lower middle incision, or transvaginally along with the specimen. An anvil can then be placed and secured with a purse string suture. The anvil and proximal sigmoid colon is then brought back into the pelvis and an end-to-end anastomosis can be performed with an end-to-end anastomosis stapler passed through the rectum. Once the device is properly activated, it is important to test the integrity of the anastomosis. This can be accomplished by clamping the proximal colon with a bowel grasper, filling the pelvis with saline, and insufflating the rectum with air while observing laparoscopically. The anastomosis can be alternatively or additionally examined by filling the rectum with indigo carmine and observing for leakage. Cystoscopy is routinely performed to ensure that there is no damage to the bladder or ureters. Ports are removed and port sites are closed in a routine manner.

In the case of recurrences that occur primarily in the upper abdomen, debulking can be performed with the robotic apparatus. In this situation, after exploratory laparoscopy and peritoneal washings are performed using conventional laparoscopy, the robot is side docked from above the patient on the right side using the same port placement as above (Fig. 1) and instrumentation is as described previously.

\section{Results}

There were 20 women that underwent 21 surgical procedures for the management of early, advanced, and recurrent ovarian, fallopian tube, or peritoneal cancer using our hybrid technique of conventional laparoscopy and RALS. There were 10 surgical procedures performed for early stage disease and 11 for advanced and/or recurrent disease (six advanced and five recurrent). The early group consisted of three cases that were stage IA (Table 1; cases 1-3), six that were stage IC (Table 1; cases 4-9), and one case that was stage IIC (Table 1, case 10). The advanced and recurrent group consisted of seven cases that were advanced, with six cases that were stage IIIC (Table 2: cases 1,2,3,5,7 and 11), one case that was stage IV (Table 2: case 4). The remainder in this group had recurrent disease (Table 2: cases $6,8,9,10$ ). There were no conversions to laparotomy in either group.

Of the 10 surgeries for early stage disease, the mean age was 42.3 years (range, 29-55), average BMI was $32.1 \mathrm{~kg} / \mathrm{m}^{2}$ (range, $17-65 \mathrm{~kg} / \mathrm{m}^{2}$ ), average estimated blood loss (EBL) was $212.5 \mathrm{ml}$ (range, $50-1,000 \mathrm{~mL}$ ), surgical time was $306.1 \mathrm{~min}$ (range, 87-639), and average length of stay (LOS) was 1.6 days (range, 1-2). In this group, the surgical procedures performed included five hysterectomies, eight oophorectomies, one cystectomy, eight omentectomies, five pelvic lymph node dissections (average, 10.3; range, 5-18), four para-aortic lymph node dissections (average, 8.6; range, 3-12), four appendectomies, and seven upper abdominal and diaphragmatic biopsies. None of these patients were upstaged after surgical and pathological staging.

There were no intraoperative complications or intraoperative transfusions, and two grade 1 postoperative complications. One patient was readmitted on postoperative day 9 with a wound infection. The other was readmitted with fever of unknown origin that resolved with IV antibiotics.

Three patients in this group underwent fertility sparing surgery (Table 1 ; cases 6,8 , and 9) [14]. In the first case listed (Table 1), the para-aortic lymphadenectomy was performed laparoscopically as there was difficulty with adequate visualization when the robotic apparatus was docked. Four patients in the early ovarian cancer group had prior surgical intervention and presented for restaging (Table 1; cases 3, 5, 8, and 9). Two patients in this group did not have an omentectomy (Table 1; cases 2 and 6). Case 2 was a double primary that was initially staged for endometrial cancer with final pathology that showed ovarian cancer as a second primary. Case 6 presented for restaging, after prior left salpingo-oophorectomy and staging. 


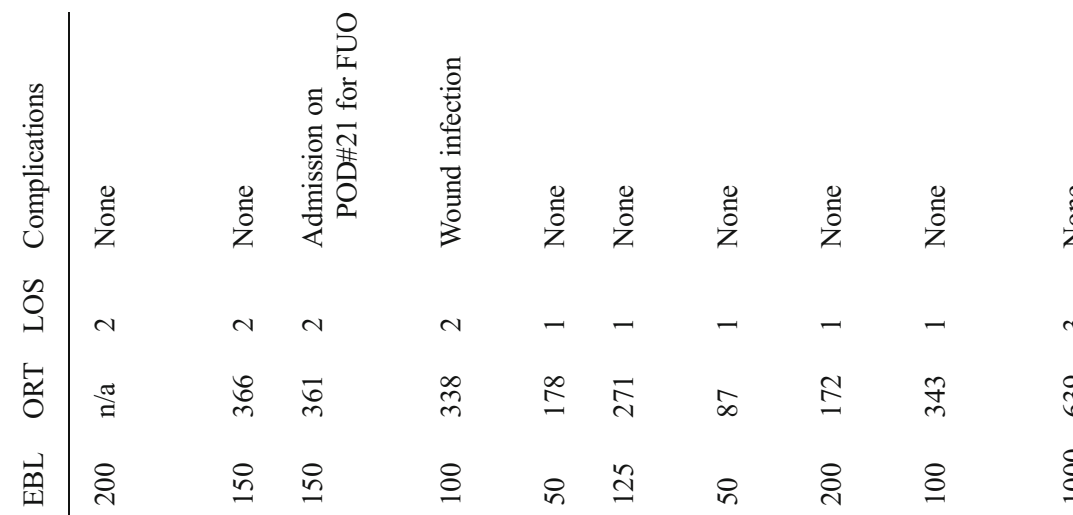

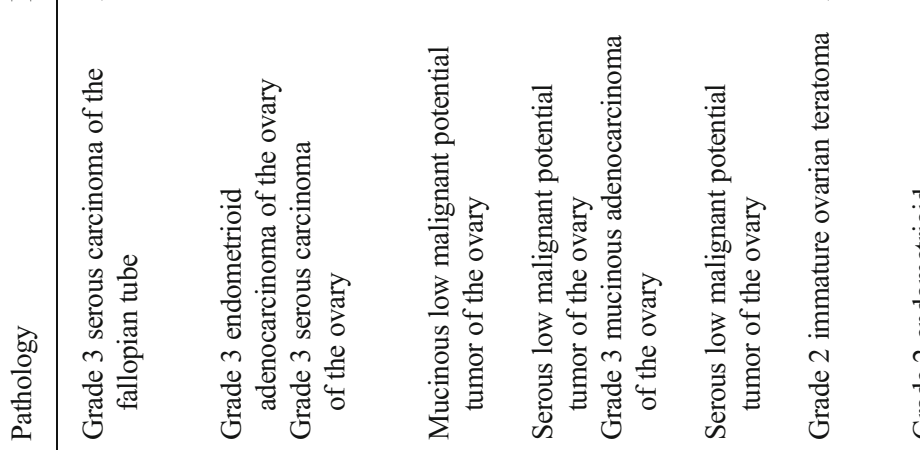

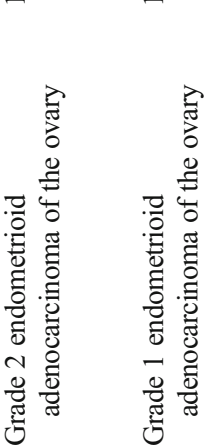

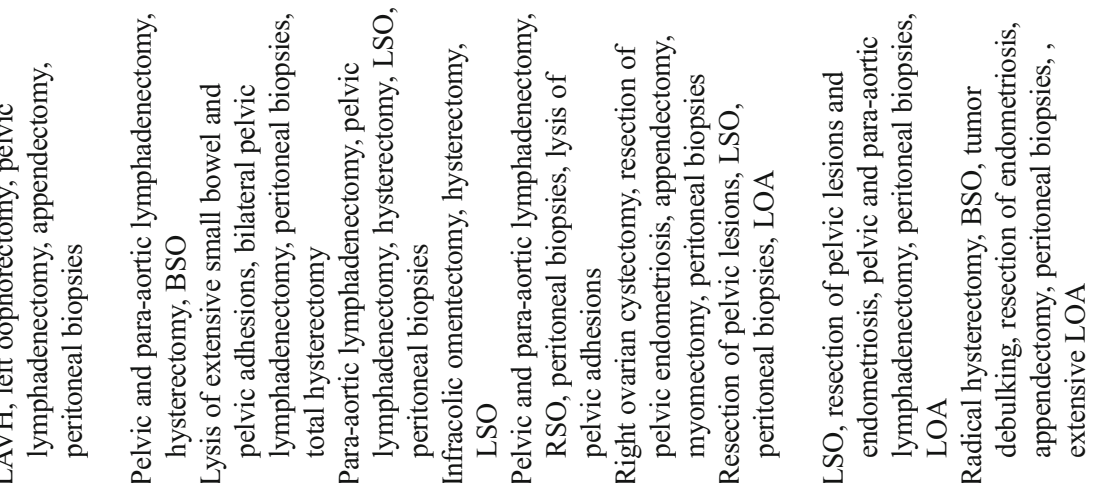

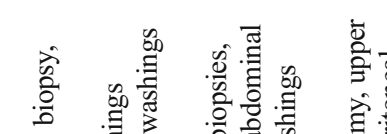

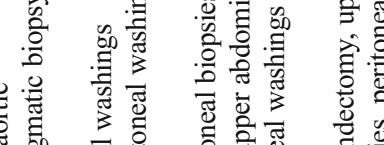

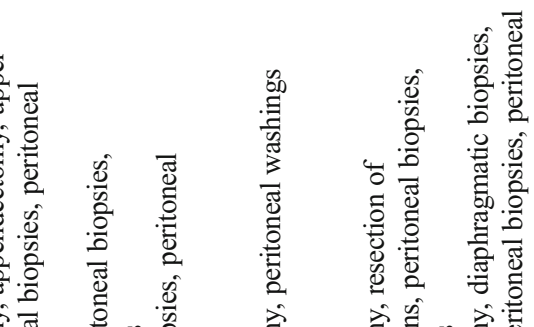

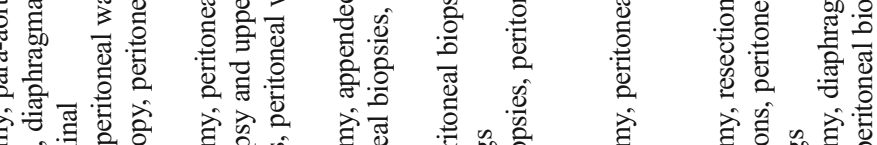

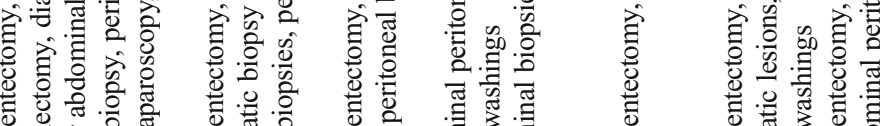

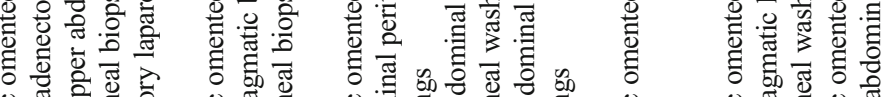

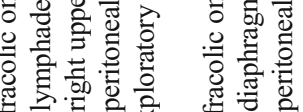

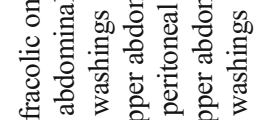

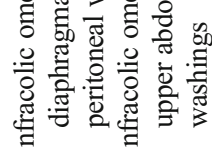

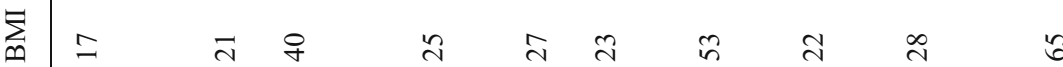

$\infty$

in

$\ln d$

$\infty$

¿

n

m

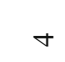

का 


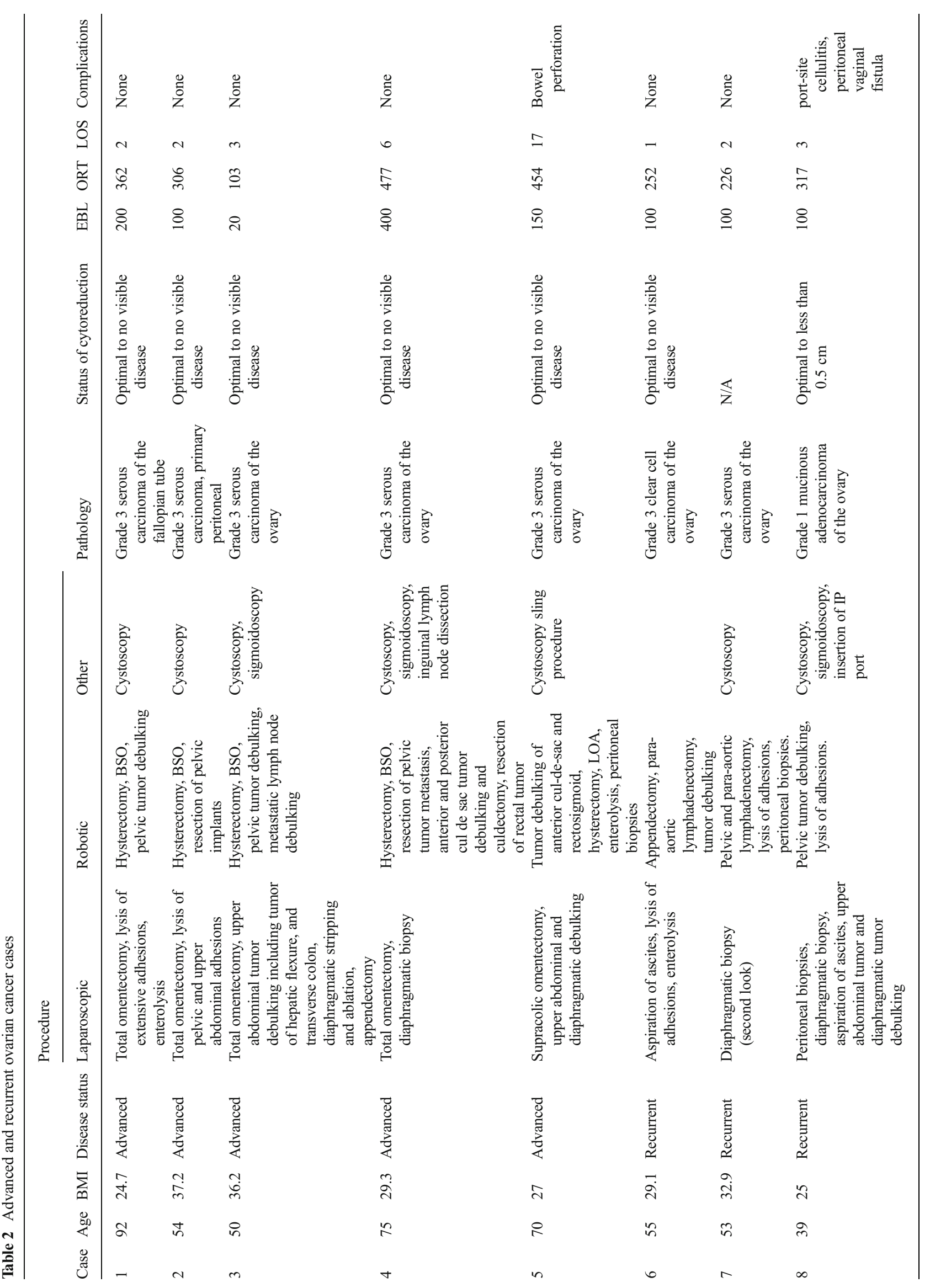


Among the 11 cases operated for advanced and/or recurrent ovarian cancer, the mean age was 63.9 years (range, 39-92), average BMI was $29.7 \mathrm{~kg} / \mathrm{m}^{2}$ (range, 22.1-37.2), EBL was $129.1 \mathrm{ml}$ (range, 20-400), operating room time was $238 \mathrm{~min}$ (range, 103-477), and LOS was 3.8 days (range, 1-17). In this group, surgical procedures consisted of six hysterectomies, five oophorectomies, eight omentectomies, three pelvic lymph node dissections (average, 10.7; range, 4-18), three para-aortic lymph node dissections (average, 5; range, 1-9), four appendectomies, five diaphragmatic biopsies, four upper abdominal debulking or diaphragmatic debulking, and one resection of a porta hepatis mass (Table 2; case 10; Fig. 4). Five patients had received neoadjuvant chemotherapy (Table 2; cases 2, 3, 4, 5, and 11). Of the 11 procedures, there was one second look (Table 2, case 7), 9 were cytoreduced to no visible disease and 1 to less than $<0.5 \mathrm{~cm}$. There were no intraoperative complications.

There were three postoperative complications; two were grade 1 and one was grade 3 . Two complications (both grade 1) occurred in the same patient; one being port-site cellulitis and the other a peritoneal vaginal fistula. Both were managed conservatively with antibiotic therapy and observation. The peritoneal vaginal fistula was revealed by leakage of peritoneal ascites. The other patient was reoperated on postoperative day 3 for a sigmoid colon perforation which required reoperation and was discharged home on day 17 (Table 2; case 5). This case was the only Intensive Care Unit admission in both early and advanced groups.

Of all the patients operated on, there were no trocar site metastases within the follow-up period. However, one patient developed trocar metastasis beyond 30 days postoperatively and had recurrent intraperitoneal disease.

\section{Discussion}

Laparoscopy offers multiple advantages over laparotomy such as better visualization, smaller incisions, shorter hospital

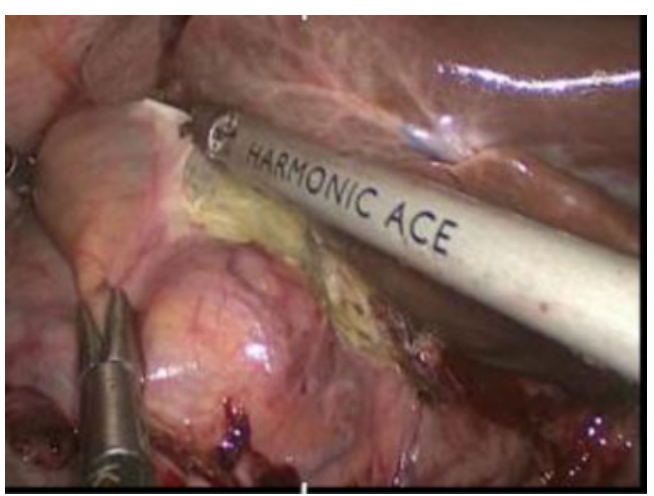

Fig. 4 Porta hepatis mass attached to the gallbladder that has been resected laparoscopically, with cholecystectomy 
stays, decreased blood loss, less need for analgesics, more rapid recovery, and shorter interval to chemotherapy and radiation when indicated. In the assessment of an adnexal mass and early-stage ovarian cancer, laparoscopy can be both diagnostic and therapeutic. The combination of laparoscopic visualization and frozen section analysis is the most reliable method for the detection of malignancy [15]. Once malignancy is diagnosed, comprehensive surgical staging can be performed laparoscopically, which has been shown to be feasible, safe, and accurate in tumors of low malignant potential and invasive early-stage disease [16-19]. Furthermore, in select cases of localized disease, it can be used to perform fertility-sparing surgical staging [14].

Minimally invasive surgery has emerged as an option in the management of advanced or recurrent ovarian cancer with multiple applications that have been presented in the literature [5, 20-22]. This includes a triage tool for resectability, primary and secondary cytoreduction, second-look evaluation, and placement of intraperitoneal catheters for chemotherapy [23].

In the early 1990s, the pioneers of laparoscopic surgery applied minimally invasive surgical techniques to gynecologic cancers for the staging of early and select cases of advanced or recurrent ovarian cancer cytoreduction [24, 25]. Since that time, the role of minimally invasive surgery in gynecologic oncology has been continuously expanding and has even been further applied to other disease sites in the female genital tract.

The advent of computer enhanced telesurgery or roboticassisted surgery has seemingly presented itself as a new alternative to conventional laparoscopy. It offers the benefits of improving the learning curve associated with conventional laparoscopy, and other additional features, such as optimal visualization and a wider range of motion for more precise surgical manipulation. Yet, this innovation currently presents with limitations in the surgical management of patients with malignancy. Conventional laparoscopy offers a better understanding of the status of the disease, while robotic-assisted laparoscopy presents as an innovation in offering precise surgical motion and visualization of the disease process. However, this comes at the cost of some key elements required with surgical management, which are possible with conventional laparoscopy.

One such limitation is the lack of haptics, which may lead to missing tumor entirely or the ability to discern between normal tissue and tissue that is involved with carcinoma. Further limitations are in accessing all four abdominal quadrants simultaneously in one surgical setting and completing comprehensive surgical evaluation and treatment for patients with ovarian malignancy. This hybrid technique is presented to overcome these challenges, and the limited manipulation that exists with robotic-assisted surgery with the current platform. This is particularly true with limitations encountered when manipulating bulky tumor and tissue, such as that of the rectosigmoid with the present robotic platform and instruments.

The hybrid technique described offers advantages that are inherent to conventional laparoscopy for management of ovarian cancer. One limitation is the need for a second setup of a conventional laparoscopy in addition to the robotic platform. In our experience, it is customary to use this setup as an adjunct to robotic-assisted surgery. Conventional laparoscopy is used for port placement, in preparation of robotic-assisted laparoscopic surgery, thus making this setup not an additional component. Thus, using this method offers an extension of the use of conventional laparoscopy, while retaining the same port setup for pelvic surgery and utilizing conventional laparoscopy to operate cephalad to the pelvis.

Another inherent benefit is that conventional laparoscopy can also be used as an initial checkpoint in triaging for resectability based on the extent of disease and surgeon experience with robotic-assisted surgery versus other conventional methods. The conventional laparoscopy setup can also be further utilized after completion of robotic-assisted surgery to survey the abdomen and pelvis for any potential injury caused by the robotic instruments not detected due to the blind spot of the robotic camera and lack of haptics. Studies are lacking with innovative methods or strategies to counter this limitation of upper abdominal access with pelvic surgery when using the robotic platform. With advanced laparoscopic skills, this method facilitates the limitations of the robotic platform. An added advantage of using this method is enhanced visualization of surgical planes that require fine dissection in the pelvis due to prior surgery or disease involvement in the pelvis. In the upper abdomen, conventional laparoscopic methods can be utilized for limited disease, with this as the initial portion of the surgery, thus reducing surgeon fatigue (of the primary surgeon).

This technique also extends to novice surgeons who are aided by tactile feedback that conventional laparoscopy extends. However, it has its limitations in more challenging skills that are more easily afforded with robotic assistance (i.e., suturing, controlling bleeding, etc.). In comparison to other described techniques for accessing the upper abdomen, this method is less cumbersome and can be used in settings with residency and fellowship training programs that have bedside assistants who are less experienced. One complication reported with use of the alternate technique of placing the robot tower at the patient's head was conversion to laparotomy in order to access bleeding from the descending branch of the inferior mesenteric artery that could not be reached by the bedside assistant or the surgeon [9]. The access afforded in operating cephalad with use of this technique is not hindered in our experience. This technique has widespread application for use, and is versatile with the level of experience required by surgical assistants. 
Minimally invasive surgical staging, more specifically cytoreductive surgery, are time-consuming procedures that are associated with surgeon fatigue that ensues with the course of the procedure. This method offers the benefit of conventional laparoscopy at the beginning of the case followed by the robotic-assisted portion of the procedure, which can help in the reduction of surgeon fatigue during the course of these lengthy procedures. This is an added benefit when the skill of an expert laparoscopist is needed for challenging initial portions of the procedure in the upper abdomen and then for more difficult portions with pelvic disease site affection in the latter portion of the procedure.

One issue that has to be addressed is the cost-effectiveness of the initial investment in the robotic platform relative to the cost of performing laparotomy, conventional laparoscopy, or robotic hybrid technique. Although only well-designed randomized studies will be able to adequately address this issue, presently, it is rather difficult to establish such a study in early and advanced ovarian cancer. In Wright et al. [26], the cost of robotic-assisted radical hysterectomy was least expensive comparative to laparotomy and even laparoscopy, due to shorter hospital stay. We believe that the use of this modality will allow more patients to be managed by a minimally invasive approach and thus reduce the number of laparotomies.

In summary, minimally invasive surgical techniques are consistently furthered from conventional laparoscopy to computer-enhanced telesurgery (also known as robotic surgery), and eventually to more compact devices that provide greater surgical precision with versatility in accessing all four abdominal quadrants more readily. Our technique serves as an interim improvisation to counter the limitations that exist with the present robotic platform.

Successful maximum cytoreductive interperitoneal metastatic disease in advanced ovarian cancer has been associated with the best outcomes [23]. However, this technique is not always possible and it carries significant morbidity. In randomized clinical trials, neoadjuvant chemotherapy followed by cytoreductive surgery has been shown much more effective in decreasing morbidity without compromising oncological outcomes [13]. Another benefit of neoadjuvant chemotherapy is that by reducing the bulky tumor, the chance of achieving optimal cytoreductive surgery by minimally invasive approach is greatly increased.

It has been our experience that using this hybrid technique, of a combination of conventional laparoscopy and the present robotic platform has been effective in the management of early, advanced, and recurrent ovarian, fallopian tube and peritoneal cancer while gaining the advantages of both technical approaches.

Conflict of interest The authors declare that there are no conflicts of interest.

\section{References}

1. Sternchos J, Finger T, Mahdavi A, Nezhat F (2013) Laparoscopic management of ovarian, fallopian tube and primary peritoneal cancer. In: Nezhat C, Nezhat F, Nezhat C (eds) Nezhat's video-assisted and robotic-assisted laparoscopy and hysteroscopy, 4th edn. Cambridge University Press, Cambridge, pp 508-525

2. Nezhat FR, Shoma D, Liu C, Chuang L, Zakashansky K (2008) Robotic radical hysterectomy versus total laparoscopic radical hysterectomy with pelvic lymphadenectomy for treatment of early cervical cancer. JSLS 12:227-237

3. Cho JE, Nezhat FR (2009) Robotics and gynecologic oncology: review of the literature. J Minim Invasive Gynecol 16(6): 669-681

4. Lim PC, Kang E, Park DH (2011) A comparative detail analysis of the learning curve and surgical outcome for robotic hysterectomy with lymphadenectomy versus laparoscopic hysterectomy with lymphadenectomy in treatment of endometrial cancer: a casematched controlled study of the first one hundred twenty-two patients. Gynecol Oncol 120:413-418

5. Magrina JF, Zanagnolo V, Noble BN et al (2011) Robotic approach for ovarian cancer: perioperative and survival results and comparison with laparoscopy and laparotomy. Gynecol Oncol 121(1):100-105

6. Ramirez PT, Adams S, Boggess JF, Burke WM, Frumovitz MM, Gardner GJ, Havrilesky LJ, Holloway R, Lowe MP, Magrina JF, Moore DH, Soliman PT, Yap S (2012) Robotic-assisted surgery in gynecologic oncology: a Society of Gynecologic Oncology consensus statement. Developed by the Society of Gynecologic Oncology's Clinical Practice Robotics Task Force. Gynecol Oncol 124(2):180 184

7. Siegel R, Deepa N, Ahmedin AJ (2012) Cancer statistics 2012. Cancer J 62(10):10-29

8. Katz VL, Lentz GM, Lobo RA, Gershenson DM (2007) Comprehensive gynecology, 5 th edn. Elsevier, Philadelphia

9. Magrina JF, Long JB, Kho RM et al (2010) Robotic transperitoneal infrarenal aortic lymphadenectomy. Int J Gynecol Cancer 20(1):184 187

10. Dindo D, Demartines N, Clavien PA (2004) Classification of surgical complications: a new proposal with evaluation in a cohort of a 6336 patients and results of a survey. Ann Surg 240: 205-213

11. Nezhat FR, Denoble SM, Cho JE, Brown DN, Soto E, Chuang L, Gretz H, Saharia P (2012) The safety and efficacy of video laparoscopic surgical debulking of recurrent ovarian, fallopian tube, and primary peritoneal cancers. JSLS 16:511518

12. Nezhat FR, DeNoble SM, Liu CS, Cho JE, Brown DN, Chuang L, Gretz H, Saharia P (2010) The safety and efficacy of laparoscopic surgical staging and debulking of apparent advanced stage ovarian, fallopian tube, and primary peritoneal cancers. JSLS 14: $155-168$

13. Vergote I, Tropè $\mathrm{C}$, Amant $\mathrm{F}$ et al (2010) Neoadjuvant chemotherapy or primary surgery in stage IIIC and IV ovarian cancer. N Engl J Med 363:943-953

14. Finger T, Nezhat F (2013) Robotic-assisted fertility sparing surgery for early ovarian cancer. JSLS (in press)

15. Nezhat F, Nezhat C, Welander CE, Benigno B (1992) Four ovarian cancers diagnosed during laparoscopic management of 1011 women with adnexal masses. Am J Obstet Gynecol 167: 790-796

16. Liu CS, Nagarsheth NP, Nezhat FR (2009) Laparoscopy and ovarian cancer: a paradigm change in the management of ovarian cancer? $\mathrm{J}$ Min Inv Gynecol 16(3):250-262 
17. Park JY, Bae J, Lim MC, Lim SY, Seo SS, Kang S et al (2008) Laparoscopic and laparotomic staging in stage I epithelial ovarian cancer: a comparison of feasibility and safety. Int J Gynecol Cancer 18:1202-1209

18. Tozzi R, Kohler C, Ferrara A, Schneider A (2004) Laparoscopic treatment of early ovarian cancer: surgical and survival outcomes. Gynecol Oncol 93:199-203

19. Nezhat FR, Ezzati M, Chuang L, Shamshirsaz AA, Rahaman J, Gretz H (2009) Laparoscopic management of early ovarian and fallopian tube cancers: surgical and survival outcome. Am J Obstet Gynecol 200(1):e1-e6

20. Nezhat FR, Datta MS, Lal N (2008) Laparoscopic cytoreduction for primary advanced or recurrent ovarian, fallopian tube, and peritoneal malignancies. Gynecol Oncol 108:S60

21. Fanning J, Yacoub E, Hojat R (2011) Laparoscopic-assisted cytoreduction for primary ovarian cancer: success, morbidity and survival. Gynecol Oncol 123:47-49
22. Krivak TC, Elkas JC, Rose GS, Sundborg M, Winter WE, Carlson J, MacKoul PJ (2005) The utility of hand-assisted laparoscopy in ovarian cancer. Gynecol Oncol 96:7276

23. Nezhat F, Lavie O (2013) The role of minimally invasive surgery in ovarian cancer. Int J Gynecol Cancer 23(5):782783

24. Querleu D, Leblanc E (1994) Laparoscopic infrarenal para-aortic lymph node dissection for restaging of carcinoma of the ovary or fallopian tube. Cancer 73:1467-1471

25. Amara DP, Nezhat C, Teng N, Nezhat F, Nezhat C, Rosati M (1996) Operative laparoscopy in the management of ovarian cancer. Surg Laparoscop Endosc 6:38-45

26. Wright JD, Herzog TJ, Neugut AI et al (2012) Comparative effectiveness of minimally invasive and abdominal radical hysterectomy for cervical cancer. Gynecol Oncol 127:1117 